\title{
Ultrasonographic study of subcutaneous penile granuloma secondary to silicone injection
}

\author{
Lucio Dell'Atti \\ Department of Urology, University Hospital "St.Anna”, Ferrara, Italy.
}

\begin{abstract}
Summary Penile augmentation has been reported in the literature by injecting various materials. This study reports our experience in management of penile augmentation complications associated with selfpenile injection of silicone liquid. After a careful ultrasound study, the penile skin was excised through a circumferential sub-coronal incision and dissected with the silicon mass. Histology was well-compatible with silicone granulomas. The patient was discharged after 24 hours. Ultrasonography has permitted preoperatively to determine if the plane between the indurated inflammatory tissue and the Buck's fascia was preserved for the complete surgical excision of affected tissue.
\end{abstract}

KEY WORDS: Penile augmentation; Complications; Silicon; Granuloma.

Submitted 14 March 2016; Accepted 8 May 2016

\section{INTRODUCTION}

A new field of male genital cosmetic surgery has developed in the past decade. The majority of men who request augmentation of their penis usually have a normally sized and functioning penis (1). However, some men want an enhancement of their penis and, therefore, use alternative filler materials injected under the skin of the genitals to increase girth, such as paraffin oil, metallic mercury, collagen, hyaluronic acid, mineral oils, and silicone (2-5). We reported a case of subcutaneous granuloma of the penis secondary to self-penile injection with silicone liquid for augmentation purposes.

\section{Case report}

A 34-years-old Caucasian male patient presented to our Urology Department with dysuria and painful swelling of the penis two months after self-penile injection with silicone for augmentation. At physical examination a tender and highly swollen penis with ulcerated tissue, especially on the ventral surface, and a considerable phimosis was seen. An ultrasonography (US) of the penis was done in the flaccid state using a 9-12 $\mathrm{MHz}$ linear array transducer. Longitudinal and transverse view of penile shaft showed diffuse thickening of the subcutaneous tissue surround the Buck's fascia with increased echogenicity (Figure 1). The corpora cavernosa and glans penis were not involved. The patient requested surgical removal of the mass. The procedure was performed under spinal anaesthesia in the supine position. The patient was catheterized for avoiding injury to the urethra. The surgical access was through a circumferential sub-coronal dorsal incision and penile skin was dissected with the silicon mass. Following excision of the mass, the corpora cavernosa were inspected for integrity. Penile skin was re-draped over the shaft and the incision was closed in one layer. The catheter was removed after 12 hours and the patient was discharged after 24 hours. Histology of the resected skin and solid tissue showed focal epidermal ulceration with dispersed areas of homogeneous basophilic material surrounded by mononuclear chronic inflammation cells, fibrosis, and micro-vesicular alteration. Pathologic examination was well-compatible with silicone granulomas.

\section{Discussion}

The literature presents scarce information on augmentation of the shaft by fillers, most in the form of case reports. Injection of fillers was in the hand of physicians, lay people, or even by the individual's own hand (3). The most common method employed in recent years for penile girth extension has been lipofilling but with disappointing results mainly because of lack an exact plane for fat injection (4). Silicone liquid injection also has been used commonly for cosmetic purposes in recent years with a wide range of indications, like correction of scars, cutaneous and subcutaneous atrophies. Silicone increases tissue bulk ideally by stimulating new collagen and fibrosis. In the case of the penis, increasing fibrosis with time could impair penile function by circumferentially compressing penile circulation (3). The first case reports of complications caused by silicone injection for penis augmentation appeared in the 1960s (5). Although silicone has been used for penile girth augmentation, as described Plaza et al. (5), it is not recommended because of many complications: swelling, penile deformities, migration and granulomatous reactions. The granulomatous reaction can further cause lymph obstruction, edema, and deformity of the penis. Other patients may present with severe infection causing multiple abscesses. There are have been few studies describing the ultrasound features of penile granulomatous reaction. US is 
Figure 1.

Longitudinal (a) and transverse (b) ultrasound B-mode view of penile shaft show diffuse thickening of the subcutaneous tissue surround the Buck's fascia with increased echogenicity (white stars); corpora cavernosa (cc) and glans penis (g) were not involved.
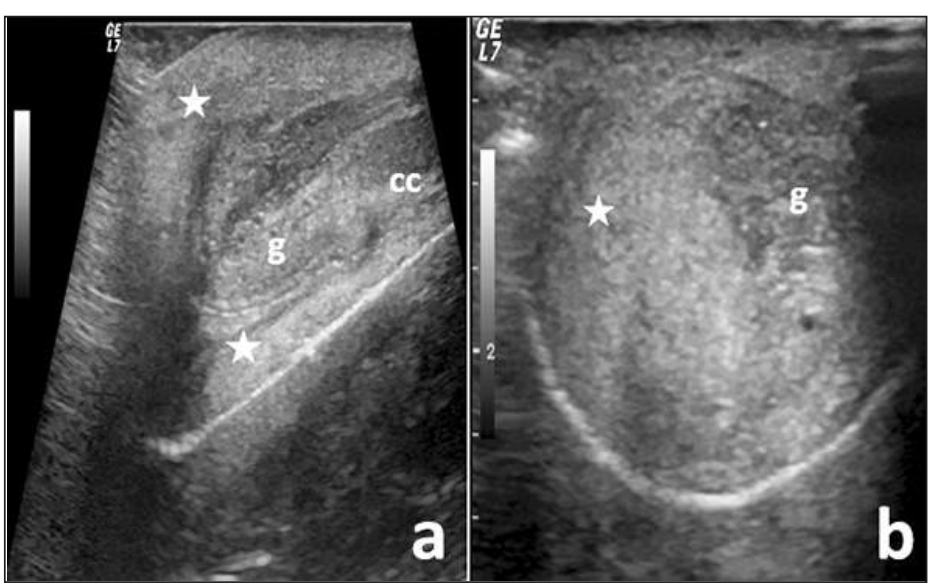

ideal in evaluating superficial structures like the penis in view of its high spatial resolution. In our case, US has permitted to determine if the plane between the indurated inflammatory tissue and the Buck's fascia was preserved for the complete surgical excision of affected tissue. It is important for the surgeons to know if there is a clear space between the foreign body material and the Buck's fascia because the complete excision of the foreign body is not possible if the Buck's fascia is involved.

In conclusion, according to the majority authors of the literature the definitive treatment for this malpractice is the complete radical excision to remove the foreign body. However, successful treatment of granuloma with oral corticosteroids has also been reported by some studies (6).

The figures of surgical treatment are posted in Supplementary materials on www.aiua.it

\section{REFERENCES}

1. Sukop A, Heracek J, Mestak O, et al. Penis augmentation by application of silicone material: complications and surgical treatment. Acta Chir Plast. 2013; 55:31-3.

2. Shaeer O, Shaeer K. Delayed complications of gel injection for penile girth augmentation. J Sex Med. 2009; 6:2072-8.

3. Silberstein J, Downs T, Goldstein I. Penile injection with silicone: case report and review of the literature. J Sex Med. 2008; 5:2231-7.

4. Inn FX, Imran FH, Ali MF, et al. Penile augmentation with result- ant foreign material granuloma and sequalae. Malays J Med Sci. 2012; 19:81-3.

5. Plaza T, Lautenschlager S. Penis swelling due to foreign body reaction after injection of silicone. J Dtsch Dermatol Ges. 2010; 8:689-91.

6. Lawrentschuk N, Angus D, Bolton DM. Sclerosing lipogranuloma of the genitalia treated with corticosteroids. Int Urol Nephrol. 2006; 38:97-9.

\section{Correspondence}

Lucio Dell'Atti, MD, PhD (Corresponding Author)

dellatti@hotmail.com

Department of Urology, University Hospital "St.Anna".

8 A. Moro Street - 44124 Cona, Ferrara, Italy 